\title{
On Jaggi-Suzuki-Type Hybrid Contraction Mappings
}

\author{
Maha Noorwali ${ }^{1}$ and Seher Sultan Yeşilkaya $\mathbb{D}^{2}$ \\ ${ }^{1}$ Department of Mathematics, Faculty of Science, King Abdulaziz University, P.O. Box 42805, Jeddah 21551, Saudi Arabia \\ ${ }^{2}$ Division of Applied Mathematics, Thu Dau Mot University, Binh Duong Province, Vietnam
}

Correspondence should be addressed to Seher Sultan Yeşilkaya; yesilkaya@tdmu.edu.vn

Received 3 August 2021; Accepted 5 October 2021; Published 20 October 2021

Academic Editor: Chi Ming Chen

Copyright (c) 2021 Maha Noorwali and Seher Sultan Yeşilkaya. This is an open access article distributed under the Creative Commons Attribution License, which permits unrestricted use, distribution, and reproduction in any medium, provided the original work is properly cited.

The aim of the paper is to introduce the concept of new hybrid contractions that combine Jaggi hybrid type contractions and Suzuki type contractions with $w$-orbital admissible. We investigate the existence and uniqueness of such new hybrid contractions in theorems and results. Further, an illustrated example is given. With the results of this study, we generalize several well-known results in the recent fixed point literature.

\section{Introduction and Preliminaries}

In the last three to four decades, metric fixed point theory was one of the crucial and useful topics of functional analysis. It has attracted the attention of researchers from several distinct disciplines. Almost a century ago, Banach [1] initiated the metric fixed point theory with a magnificently simple but enormously useful result, known as the "Banach contraction principle." It is one of the useful theorems for solving differential integral equations to guarantee both the existence and the uniqueness of the solution. A wide variety of problems arising in different areas of pure and applied mathematics, such as differential equations, discrete and continuous dynamical systems, and nonlinear analysis, can be modeled as fixed point equations of the form $u=\mathrm{E} u$. Soon afterward, the Banach contraction principle was extended by Caccioppoli [2] to the setting of a complete metric space, which states that, in a complete metric space $(\mathscr{X}, \mathrm{d})$, any Banach contraction $\mathrm{E}: \mathscr{X} \longrightarrow \mathscr{X}$ is a Picard operator; we refer to it as the "Banach-Caccioppoli theorem." These main theorems have been generalized in different directions by many researchers. Among all, the authors observed a very general result in a general setting by using a simulation function. For more interesting results, see, e.g., $[3,4]$.

Jaggi [5] demonstrated the following theorem satisfying a contractive condition of a rational type.
Theorem 1 (see [5]). Let $E$ be a continuous self-map defined on complete metric space $(\mathscr{X}, d)$. Further, let E satisfy the following condition:

$$
d(E u, E z) \leq \alpha \frac{d(u, E u) d(z, E z)}{d(u, z)}+\beta d(u, z),
$$

for all distinct points $u, z \in \mathscr{X}$ and where $\alpha, \beta \in[0,1)$ with $\alpha$ $+\beta<1$. Then, E has a unique fixed point in $\mathscr{X}$.

Very recently in 2018, Karapinar [6] obtained a new interesting type of contraction generalized from the wellknown Kannan contraction by adopting an interpolative approach. In [7], a common fixed point of the interpolative Kannan contraction was considered.

Karapinar's definition and theorem are listed as follows.

Definition 2 (see [6]). Let $(\mathscr{X}, d)$ be a metric space. A selfmapping $E: \mathscr{X} \longrightarrow \mathscr{X}$ is said to be an interpolative Kannan-type contraction if there are two constants $\lambda \in[0,1)$ and $\alpha \in(0,1)$ such that

$$
\mathrm{d}(\mathrm{E} u, \mathrm{E} z) \leq \lambda[\mathrm{d}(u, \mathrm{E} u)]^{\alpha} \cdot[\mathrm{d}(z, \mathrm{E} z)]^{1-\alpha},
$$

for all $u, z \in \mathscr{X}$ with $u \neq \mathrm{E} u$. 
Theorem 3 (see [6]). Let $(\mathscr{X}, d)$ be a complete metric space and $E: X \longrightarrow X$ be an interpolative Kannan-type contraction. Then, E has a unique fixed point in $\mathscr{X}$.

Recall the notion of $\alpha$-admissibility introduced by Samet et al. [8].

Definition 4. A mapping E : $\mathscr{X} \longrightarrow \mathscr{X}$ is called $\alpha$-admissible if, for all $u, z \in \mathscr{X}$, we have

$$
\alpha(u, z) \geq 1 \Rightarrow \alpha(\mathrm{E} u, \mathrm{E} z) \geq 1
$$

where $\alpha: \mathscr{X} \times \mathscr{X} \longrightarrow[0, \infty)$ is a given function.

Afterward, as a modification in the concept of $\alpha$-admissible maps, Popescu [9] introduced $w$-orbital admissible maps.

Definition 5. Let $w: \mathscr{X} \times \mathscr{X} \longrightarrow[0, \infty)$ be a mapping and $\mathscr{X} \neq \varnothing$. A self-mapping $\mathrm{E}: \mathscr{X} \longrightarrow \mathscr{X}$ is called $w$-orbital admissible if, for all $u \in \mathscr{X}$, we have

$$
w(u, \mathrm{E} u) \geq 1 \Rightarrow w\left(\mathrm{E} u, \mathrm{E}^{2} u\right) \geq 1
$$

The following condition has often been considered in order to avoid the continuity of the involved contractive mappings.

(R) A space $(\mathscr{X}, \mathrm{d})$ is called $w$-regular, if whenever $\left\{u_{\mathrm{m}}\right\}$ is a sequence in $\mathscr{X}$ such that $\alpha\left(u_{\mathrm{m}}, u_{\mathrm{m}+1}\right) \geq 1$ for all $\mathrm{m}$ and $u_{\mathrm{m}} \longrightarrow u \in \mathscr{X}$ as $\mathrm{m} \longrightarrow \infty$, then there exists a subsequence $\left\{u_{\mathrm{m}(k)}\right\}$ of $\left\{u_{\mathrm{m}}\right\}$ such that $w\left(u_{\mathrm{m}(k)}, u\right) \geq 1$ for all $k$.

In 2008, Suzuki [10] published one of the most comprehensive generalizations of Banach's and Edelstein's basic results. Suzuki contraction is when the contractive condition required to satisfy is not for all points of the domain. The existence and uniqueness of fixed points of maps satisfying a Suzuki type contraction has been extensively studied (see [11-15]). Later, Popescu [9] modified the nonexpansiveness situation with the weaker $C$-condition presented by Suzuki. The $C$-condition is defined as follows.
Definition 6. A mapping $\mathrm{E}$ on a metric space $(\mathscr{X}, d)$ satisfies the $C$-condition if

$$
\frac{1}{2} \mathrm{~d}(u, \mathrm{E} u) \leq \mathrm{d}(u, z) \Rightarrow \mathrm{d}(\mathrm{E} u, \mathrm{E} z) \leq \mathrm{d}(u, z),
$$

for each $u, z \in \mathscr{X}$.

Recently, Mitrovic et al. [16] used interpolation contraction and Reich contraction together and combined these two contractions in $b$-metric spaces. The combination of these two types of contractions has been called the new hybrid type contraction. In the last years, inspired by the result in [6], Karapinar and Fulga [17] introduced a new hybridtype contraction that combines Jaggi type contractions and interpolative-type contractions in the framework of complete metric spaces. In this paper, we investigate the JaggiSuzuki type hybrid contraction, inspired by the new contraction in [17], which is a combination of Jaggi hybrid type contractions and Suzuki type contractions with $w$-orbital admissible in the framework of complete metric spaces. We introduce the existence and uniqueness of a fixed point for this contraction.

\section{Main Results}

We start by recalling the definition of the family $\Psi$ as the set of all nondecreasing self-mappings $\psi$ on $[0, \infty)$ such that $\sum_{\mathrm{m}=1}^{\infty} \psi^{\mathrm{m}}(v)<\infty$ for every $v>0$. Notice that for $\psi \in \Psi$, we have $\psi(0)=0$ and $\psi(v)<v$ for all $v>0$ (see [18]).

Here are our main definition and theorem.

Definition 7 . Let $(\mathscr{X}, d)$ be a metric space. We say that the mapping $\mathrm{E}: \mathscr{X} \longrightarrow \mathscr{X}$ is a Jaggi-Suzuki type hybrid contraction if there exist $\psi \in \Psi$ and $w: \mathscr{X} \times \mathscr{X} \longrightarrow[0, \infty)$ such that

$\frac{1}{2} \mathrm{~d}(u, \mathrm{E} u) \leq \mathrm{d}(u, z) \Rightarrow w(u, z) \mathrm{d}(\mathrm{E} u, \mathrm{E} z) \leq \psi\left(\mu_{\mathrm{E}}^{s}(u, z)\right)$,

for each $u, z \in \mathscr{X}$ where $s \geq 0$ and $\rho_{i} \geq 0, i=1,2$, such that $\rho_{1}+\rho_{2}=1$ with $\rho_{1}<1 / 2$,

$$
\mu_{\mathrm{E}}^{s}(u, z)=\left\{\begin{array}{l}
{\left[\rho_{1}\left(\frac{\mathrm{d}(u, \mathrm{E} u) \mathrm{d}(z, \mathrm{E} z)}{d(u, z)}\right)^{s}+\rho_{2}(\mathrm{~d}(u, z))^{s}\right]^{1 / s}, \quad \text { for } s>0, u \neq z,} \\
(\mathrm{~d}(u, \mathrm{E} u))^{\rho_{1}}(\mathrm{~d}(z, \mathrm{E} z))^{\rho_{2}}, \quad \text { for } s=0, u, z \in \mathscr{X} \backslash \operatorname{Fix}(\mathrm{E}) .
\end{array}\right.
$$

Theorem 8. Let $(\mathscr{X}, d)$ be a complete metric space and $E: \mathscr{X}$ $\longrightarrow \mathscr{X}$ be a Jaggi-Suzuki-type hybrid contraction. Assume also that $E$ is $w$-orbital admissible mapping and $w\left(u_{0}, E u_{0}\right) \geq 1$ for some $u_{0} \in \mathscr{X}$. Then, $E$ has a fixed point in $\mathscr{X}$ provided that at least one of the following conditions holds:

$\left(j_{1}\right)(\mathscr{X}, d)$ is $w$-regular

$\left(j_{2}\right) E$ is continuous

$\left(j_{3}\right) E^{2}$ is continuous and $w(u, E u) \geq 1$ where $u \in \operatorname{Fix}\left(E^{2}\right)$
Proof. Let the sequence $\left\{u_{\mathrm{m}}\right\}$ in $\mathscr{X}$ be constructed by $\mathrm{E}^{\mathrm{m}}($ $\left.u_{0}\right)=u_{\mathrm{m}}$ for all positive integer $\mathrm{m}$, where $u_{0} \in \mathscr{X}$ such that $w\left(u_{0}, \mathrm{E} u_{0}\right) \geq 1$.

If $u_{\mathrm{m}_{0}}=u_{\mathrm{m}_{0+1}}$ for some integer $\mathrm{m}_{0}$, then $u_{\mathrm{m}_{0}}$ is a fixed point of $\mathrm{E}$, so we shall assume that $u_{\mathrm{m}} \neq u_{\mathrm{m}+1}$ for all positive integer $\mathrm{m}$. Since $\mathrm{E}$ is $w$-orbital admissible, so $w\left(u_{0}, \mathrm{E} u_{0}\right)=$ $w\left(u_{0}, u_{1}\right) \geq 1$ implies that $w\left(u_{1}, \mathrm{E} u_{1}\right)=w\left(u_{1}, u_{2}\right) \geq 1$. Continuing this argument, we get 


$$
w\left(u_{\mathrm{m}}, u_{\mathrm{m}+1}\right) \geq 1
$$

Case 1. $s>0$, by taking $\mu_{\mathrm{E}}^{s}(u, z)$ choosing $u=u_{\mathrm{m}-1}$ and $z=$ $\mathrm{E} u_{\mathrm{m}-1}=u_{\mathrm{m}}$ in (6), we get

$$
\begin{aligned}
\frac{1}{2} \mathrm{~d}\left(u_{\mathrm{m}-1}, \mathrm{E} u_{\mathrm{m}-1}\right) & =\frac{1}{2} \mathrm{~d}\left(u_{\mathrm{m}-1}, u_{\mathrm{m}}\right) \leq \mathrm{d}\left(u_{\mathrm{m}-1}, u_{\mathrm{m}}\right) \\
& \Rightarrow w\left(u_{\mathrm{m}-1}, u_{\mathrm{m}}\right) \mathrm{d}\left(\mathrm{E} u_{\mathrm{m}-1}, \mathrm{E} u_{\mathrm{m}}\right) \\
& \leq \psi\left(\mu_{\mathrm{E}}^{\mathrm{s}}\left(u_{\mathrm{m}-1}, u_{\mathrm{m}}\right)\right),
\end{aligned}
$$

where

$$
\begin{aligned}
\mu_{\mathrm{E}}^{s} & \left.u_{\mathrm{m}-1}, \mathrm{E} u_{\mathrm{m}-1}\right) \\
= & {\left[\rho_{1}\left(\frac{\mathrm{d}\left(u_{\mathrm{m}-1}, \mathrm{E} u_{\mathrm{m}-1}\right) \mathrm{d}\left(\mathrm{E} u_{\mathrm{m}-1}, \mathrm{E}^{2} u_{\mathrm{m}-1}\right)}{\mathrm{d}\left(u_{\mathrm{m}-1}, \mathrm{E} u_{\mathrm{m}-1}\right)}\right)^{s}\right.} \\
& \left.+\rho_{2}\left(\mathrm{~d}\left(u_{\mathrm{m}-1}, \mathrm{E} u_{\mathrm{m}-1}\right)\right)^{s}\right]^{1 / s} \\
= & {\left[\rho_{1}\left(\frac{\mathrm{d}\left(u_{\mathrm{m}-1}, u_{\mathrm{m}}\right) \mathrm{d}\left(u_{\mathrm{m}}, u_{\mathrm{m}+1}\right)}{\mathrm{d}\left(u_{\mathrm{m}-1}, u_{\mathrm{m}}\right)}\right)^{s}+\rho_{2}\left(\mathrm{~d}\left(u_{\mathrm{m}-1}, u_{\mathrm{m}}\right)\right)^{s}\right]^{1 / s} } \\
= & {\left[\rho_{1}\left(\mathrm{~d}\left(u_{\mathrm{m}}, u_{\mathrm{m}+1}\right)\right)^{s}+\rho_{2}\left(\mathrm{~d}\left(u_{\mathrm{m}-1}, u_{\mathrm{m}}\right)\right)^{s}\right]^{1 / s} . }
\end{aligned}
$$

Hereafter, we find that

$$
\begin{aligned}
\mathrm{d}\left(u_{\mathrm{m}}, u_{\mathrm{m}+1}\right) & \leq w\left(u_{\mathrm{m}-1}, u_{\mathrm{m}}\right) \mathrm{d}\left(\mathrm{E} u_{\mathrm{m}-1}, \mathrm{E} u_{\mathrm{m}}\right) \\
& \leq \psi\left(\mu_{\mathrm{E}}^{s}\left(u_{\mathrm{m}-1}, u_{\mathrm{m}}\right)\right) \\
& =\psi\left(\left[\rho_{1}\left(\mathrm{~d}\left(u_{\mathrm{m}}, u_{\mathrm{m}+1}\right)\right)^{s}+\rho_{2}\left(\mathrm{~d}\left(u_{\mathrm{m}-1}, u_{\mathrm{m}}\right)\right)^{s}\right]^{1 / s}\right) .
\end{aligned}
$$

Suppose that $\mathrm{d}\left(u_{\mathrm{m}}, u_{\mathrm{m}+1}\right) \geq \mathrm{d}\left(u_{\mathrm{m}-1}, u_{\mathrm{m}}\right)$, so, using (11), we write

$$
\begin{aligned}
\mathrm{d}\left(u_{\mathrm{m}}, u_{\mathrm{m}+1}\right) & \leq \psi\left(\left[\rho_{1}\left(\mathrm{~d}\left(u_{\mathrm{m}}, u_{\mathrm{m}+1}\right)\right)^{s}+\rho_{2}\left(\mathrm{~d}\left(u_{\mathrm{m}-1}, u_{\mathrm{m}}\right)\right)^{s}\right]^{\frac{1}{s}}\right) \\
& \leq \psi\left(\left[\rho_{1}\left(\mathrm{~d}\left(u_{\mathrm{m}}, u_{\mathrm{m}+1}\right)\right)^{s}+\rho_{2}\left(\mathrm{~d}\left(u_{\mathrm{m}}, u_{\mathrm{m}+1}\right)\right)^{s}\right]^{\frac{1}{s}}\right) \\
& \leq \psi\left(\left[\left(\rho_{1}+\rho_{2}\right)\left(\mathrm{d}\left(u_{\mathrm{m}}, u_{\mathrm{m}+1}\right)\right)^{s}\right]^{\frac{1}{s}}\right) \\
& =\psi\left(\left[\left(\mathrm{d}\left(u_{\mathrm{m}}, u_{\mathrm{m}+1}\right)\right)^{s}\right]^{\frac{1}{s}}\right) \\
& =\psi\left(\mathrm{d}\left(u_{\mathrm{m}}, u_{\mathrm{m}+1}\right)\right)<\mathrm{d}\left(u_{\mathrm{m}}, u_{\mathrm{m}+1}\right),
\end{aligned}
$$

which is a contradiction. Then, we get

$$
\mathrm{d}\left(u_{\mathrm{m}}, u_{\mathrm{m}+1}\right)<\mathrm{d}\left(u_{\mathrm{m}-1}, u_{\mathrm{m}}\right) .
$$

Eventually, from (11), we have

$$
\left.\mathrm{d}\left(u_{\mathrm{m}}, u_{\mathrm{m}+1}\right)\right) \leq \psi\left(\mathrm{d}\left(u_{\mathrm{m}-1}, u_{\mathrm{m}}\right)\right)<\mathrm{d}\left(u_{\mathrm{m}-1}, u_{\mathrm{m}}\right),
$$

and by repeating this process, we find that

$$
\mathrm{d}\left(u_{\mathrm{m}}, u_{\mathrm{m}+1}\right) \leq \psi^{\mathrm{m}}\left(\mathrm{d}\left(u_{0}, u_{1}\right)\right),
$$

for any $\mathrm{m} \in \mathbb{N}$. We claim that $\left\{u_{\mathrm{m}}\right\}$ is a Cauchy sequence in $(\mathscr{X}, \mathrm{d})$. Then, using the triangle inequality with (15), we can write

$$
\begin{aligned}
\mathrm{d}\left(u_{\mathrm{m}}, u_{\mathrm{m}+l}\right) \leq & \mathrm{d}\left(u_{\mathrm{m}}, u_{\mathrm{m}+1}\right)+\mathrm{d}\left(u_{\mathrm{m}+1}, u_{\mathrm{m}+2}\right)+\cdots \\
& +\mathrm{d}\left(u_{\mathrm{m}+l-1}, u_{\mathrm{m}+l}\right) \leq \psi^{\mathrm{m}} \mathrm{d}\left(u_{0}, u_{1}\right) \\
& +\psi^{\mathrm{m}+1}\left(\mathrm{~d}\left(u_{0}, u_{1}\right)\right)+\cdots+\psi^{\mathrm{m}+l-1}\left(\mathrm{~d}\left(u_{0}, u_{1}\right)\right) \\
= & \sum_{k=\mathrm{m}}^{\mathrm{m}+l-1} \psi^{k}\left(\mathrm{~d}\left(u_{0}, u_{1}\right)=S_{\mathrm{m}+l-1}-S_{\mathrm{m}-1},\right.
\end{aligned}
$$

where $S_{t}=\sum_{k=0}^{t} \psi^{k}\left(\mathrm{~d}\left(u_{0}, u_{1}\right)\right)$. But, $\psi \in \Psi$, so the series $\sum_{k=0}^{\infty} \psi^{k}\left(\mathrm{~d}\left(u_{0}, u_{1}\right)\right)$ is convergent, then there exists a positive real number $S$ such that $\lim _{t \rightarrow \infty} S_{t}=S$. As a result, letting $\mathrm{m}, l \longrightarrow \infty$ in the above inequality, we obtain

$$
\mathrm{d}\left(u_{\mathrm{m}}, u_{\mathrm{m}+l}\right) \longrightarrow 0
$$

Thus, $\left\{u_{\mathrm{m}}\right\}$ is a Cauccess of the space $(\mathscr{X}, \mathrm{d})$; it follows that there exists $v \in \mathscr{X}$ such that

$$
\lim _{\mathrm{m} \longrightarrow \infty} \mathrm{d}\left(u_{\mathrm{m}}, v\right)=0
$$

We assert that $v$ is a fixed point of $\mathrm{E}$.

If the assumption $\left(j_{1}\right)$ holds, we get $w\left(u_{\mathrm{m}}, v\right) \geq 1$, and we assert that

$$
\text { either } \begin{aligned}
\frac{1}{2} \mathrm{~d}\left(u_{\mathrm{m}}, \mathrm{E} u_{\mathrm{m}}\right) & \leq \mathrm{d}\left(u_{\mathrm{m}}, v\right) \text { or } \frac{1}{2} \mathrm{~d}\left(\mathrm{E} u_{\mathrm{m}}, \mathrm{E}\left(\mathrm{E} u_{\mathrm{m}}\right)\right) \\
& \leq \mathrm{d}\left(\mathrm{E} u_{\mathrm{m}}, v\right)
\end{aligned}
$$

for all $m \in \mathbb{N}$. Since, if we suppose that

$$
\frac{1}{2} \mathrm{~d}\left(u_{\mathrm{m}}, \mathrm{E} u_{\mathrm{m}}\right)>\mathrm{d}\left(u_{\mathrm{m}}, v\right), \frac{1}{2} \mathrm{~d}\left(\mathrm{E} u_{\mathrm{m}}, \mathrm{E}\left(\mathrm{E} u_{\mathrm{m}}\right)\right)>\mathrm{d}\left(\mathrm{E} u_{\mathrm{m}}, v\right),
$$

then, taking into account the triangle inequality and the fact that the sequence $\left\{\mathrm{d}\left(u_{\mathrm{m}}, u_{\mathrm{m}+1}\right)\right\}$ is decreasing, we obtain that

$$
\begin{aligned}
\mathrm{d}\left(u_{\mathrm{m}}, u_{\mathrm{m}+1}\right) & =\mathrm{d}\left(u_{\mathrm{m}}, \mathrm{E} u_{\mathrm{m}}\right) \leq \mathrm{d}\left(u_{\mathrm{m}}, v\right)+\mathrm{d}\left(v, \mathrm{E} u_{\mathrm{m}}\right) \\
& <\frac{1}{2} \mathrm{~d}\left(u_{\mathrm{m}}, \mathrm{E} u_{\mathrm{m}}\right)+\frac{1}{2} \mathrm{~d}\left(\mathrm{E} u_{\mathrm{m}}, \mathrm{E}\left(\mathrm{E} u_{\mathrm{m}}\right)\right) \\
& =\frac{1}{2} \mathrm{~d}\left(u_{\mathrm{m}}, u_{\mathrm{m}+1}\right)+\frac{1}{2} \mathrm{~d}\left(u_{\mathrm{m}+1}, u_{\mathrm{m}+2}\right) \\
& \leq \frac{1}{2} \mathrm{~d}\left(u_{\mathrm{m}}, u_{\mathrm{m}+1}\right)+\frac{1}{2} \mathrm{~d}\left(u_{\mathrm{m}}, u_{\mathrm{m}+1}\right) \\
& =\mathrm{d}\left(u_{\mathrm{m}}, u_{\mathrm{m}+1}\right),
\end{aligned}
$$


a contradiction. Thus, for all $m \in \mathbb{N}$, either

$$
\frac{1}{2} \mathrm{~d}\left(u_{\mathrm{m}}, \mathrm{E} u_{\mathrm{m}}\right) \leq \mathrm{d}\left(u_{\mathrm{m}}, v\right)
$$

or

$$
\frac{1}{2} \mathrm{~d}\left(\mathrm{E} u_{\mathrm{m}}, \mathrm{E}\left(\mathrm{E} u_{\mathrm{m}}\right)\right) \leq \mathrm{d}\left(\mathrm{E} u_{\mathrm{m}}, v\right)
$$

holds. In case that (22) holds, we get

$$
\begin{aligned}
\mathrm{d}\left(u_{\mathrm{m}+1}, \mathrm{E} v\right) & \leq w\left(u_{\mathrm{m}}, v\right) \mathrm{d}\left(\mathrm{E} u_{\mathrm{m}}, \mathrm{E} v\right) \\
& \leq \psi\left[\rho_{1}\left(\frac{\mathrm{d}\left(u_{\mathrm{m}}, \mathrm{E} u_{\mathrm{m}}\right) d(v, \mathrm{E} v)}{d\left(u_{\mathrm{m}}, v\right)}\right)^{s}+\rho_{2}\left(d\left(u_{\mathrm{m}}, v\right)\right)^{s}\right]^{1 / s} \\
& <\left[\rho_{1}\left(\frac{\mathrm{d}\left(u_{\mathrm{m}}, \mathrm{E} u_{\mathrm{m}}\right) \mathrm{d}(v, \mathrm{E} v)}{d\left(u_{\mathrm{m}}, v\right)}\right)^{s}+\rho_{2}\left(\mathrm{~d}\left(u_{\mathrm{m}}, v\right)\right)^{s}\right]^{1 / s} \\
& =\left[\rho_{1}\left(\frac{\mathrm{d}\left(u_{\mathrm{m}}, u_{\mathrm{m}+1}\right) \mathrm{d}(v, \mathrm{E} v)}{d\left(u_{\mathrm{m}}, v\right)}\right)^{s}+\rho_{2}\left(\mathrm{~d}\left(u_{\mathrm{m}}, v\right)\right)^{s}\right]^{1 / s} \\
& \leq\left[\rho_{1}\left(\frac{2 \mathrm{~d}\left(u_{\mathrm{m}}, v\right) \mathrm{d}(v, \mathrm{E} v)}{d\left(u_{\mathrm{m}}, v\right)}\right)^{s}+\rho_{2}\left(\mathrm{~d}\left(u_{\mathrm{m}}, v\right)\right)^{s}\right]^{1 / s} .
\end{aligned}
$$

If the second condition, (23), holds, we can write

$$
\begin{aligned}
\mathrm{d}\left(u_{\mathrm{m}+2}, \mathrm{E} v\right) \\
\quad \leq w\left(u_{\mathrm{m}+1}, v\right) \mathrm{d}\left(\mathrm{E}^{2} u_{\mathrm{m}}, \mathrm{E} v\right) \\
\quad \leq \psi\left[\rho_{1}\left(\frac{\mathrm{d}\left(\mathrm{E} u_{\mathrm{m}}, \mathrm{E}^{2} u_{\mathrm{m}}\right) \mathrm{d}(v, \mathrm{E} v)}{\mathrm{d}\left(\mathrm{E} u_{\mathrm{m}}, v\right)}\right)^{s}+\rho_{2}\left(\mathrm{~d}\left(\mathrm{E} u_{\mathrm{m}}, v\right)\right)^{s}\right]^{1 / s} \\
\quad<\left[\rho_{1}\left(\frac{\mathrm{d}\left(\mathrm{E} u_{\mathrm{m}}, \mathrm{E}^{2} u_{\mathrm{m}}\right) \mathrm{d}(v, \mathrm{E} v)}{\mathrm{d}\left(\mathrm{E} u_{\mathrm{m}}, v\right)}\right)^{s}+\rho_{2}\left(\mathrm{~d}\left(\mathrm{E} u_{\mathrm{m}}, v\right)\right)^{s}\right]^{1 / s} \\
\quad=\left[\rho_{1}\left(\frac{\mathrm{d}\left(u_{\mathrm{m}+1}, u_{\mathrm{m}+2}\right) \mathrm{d}(v, \mathrm{E} v)}{\mathrm{d}\left(u_{\mathrm{m}+1}, v\right)}\right)^{s}+\rho_{2}\left(\mathrm{~d}\left(u_{\mathrm{m}+1}, v\right)\right)^{s}\right]^{1 / s} \\
\quad \leq\left[\rho_{1}\left(\frac{2 \mathrm{~d}\left(u_{\mathrm{m}+1}, v\right) \mathrm{d}(v, \mathrm{E} v)}{\mathrm{d}\left(u_{\mathrm{m}+1}, v\right)}\right)^{s}+\rho_{2}\left(\mathrm{~d}\left(u_{\mathrm{m}+1}, v\right)\right)^{s}\right]^{1 / s} .
\end{aligned}
$$

Hence, taking $\mathrm{m} \longrightarrow \infty$ in (24) and (25),

$$
\mathrm{d}(v, \mathrm{E} v)<2 \rho_{1}^{1 / s} \mathrm{~d}(v, \mathrm{E} v) \leq \mathrm{d}(v, \mathrm{E} v),
$$

which is a contraction. Therefore, we get that $\mathrm{d}(v, \mathrm{E} v)=0$, that is, $v=\mathrm{E} v$.

If the assumption $\left(j_{2}\right)$ is true, that is, the mapping $\mathrm{E}$ is continuous, we obtain

$$
\mathrm{E} v=\lim _{\mathrm{m} \longrightarrow \infty} \mathrm{E} u_{\mathrm{m}}=\lim _{\mathrm{m} \longrightarrow \infty} u_{\mathrm{m}+1}=v .
$$

In case that last assumption $\left(j_{3}\right)$ holds, from above, we get $\mathrm{E}^{2} v=\lim _{\mathrm{m} \longrightarrow \infty} \mathrm{E}^{2} u_{\mathrm{m}}=\lim _{\mathrm{m} \longrightarrow \infty} u_{\mathrm{m}+2}=v$ and we aim to prove that also $\mathrm{E} v=v$. Assuming on the contrary that $v \neq \mathrm{E}$ $v$, from

$$
\frac{1}{2} \mathrm{~d}\left(\mathrm{E} v, \mathrm{E}^{2} v\right)=\frac{1}{2} \mathrm{~d}(\mathrm{E} v, v) \leq \mathrm{d}(\mathrm{E} v, v)
$$

using (6), we obtain that

$$
\begin{aligned}
\mathrm{d}(v, \mathrm{E} v) & =\mathrm{d}\left(\mathrm{E}^{2} v, \mathrm{E} v\right) \leq w(\mathrm{E} v, v) \mathrm{d}\left(\mathrm{E}^{2} v, \mathrm{E} v\right) \\
& \leq \psi\left[\rho_{1}\left(\frac{\mathrm{d}\left(\mathrm{E} v, \mathrm{E}^{2} v\right) \mathrm{d}(v, \mathrm{E} v)}{\mathrm{d}(\mathrm{E} v, v)}\right)^{s}+\rho_{2}(\mathrm{~d}(\mathrm{E} v, v))^{s}\right]^{1 / s} \\
& <\left[\rho_{1}\left(\frac{\mathrm{d}\left(\mathrm{E} v, \mathrm{E}^{2} v\right) \mathrm{d}(v, \mathrm{E} v)}{\mathrm{d}(\mathrm{E} v, v)}\right)^{s}+\rho_{2}(\mathrm{~d}(\mathrm{E} v, v))^{s}\right]^{1 / s} \\
& =\mathrm{d}(v, \mathrm{E} v),
\end{aligned}
$$

a contradiction. As a result, $v=\mathrm{E} v$, that is, $v$ is a fixed point of the mapping $\mathrm{E}$.

Case 2. $s=0$, by taking $\mu_{\mathrm{E}}^{s}(u, z)$ choosing $u=u_{\mathrm{m}-1}$ and $z=$ $\mathrm{E} u_{\mathrm{m}-1}=u_{\mathrm{m}}$ in (6), we obtain

$$
\begin{aligned}
\frac{1}{2} \mathrm{~d}\left(u_{\mathrm{m}-1}, \mathrm{E} u_{\mathrm{m}-1}\right)=\frac{1}{2} \mathrm{~d}\left(u_{\mathrm{m}-1}, u_{\mathrm{m}}\right) \leq \mathrm{d}\left(u_{\mathrm{m}-1}, u_{\mathrm{m}}\right) \Rightarrow \\
\begin{aligned}
\mathrm{d}\left(u_{\mathrm{m}}, u_{\mathrm{m}+1}\right) \leq & w\left(u_{\mathrm{m}-1}, u_{\mathrm{m}}\right) \mathrm{d}\left(\mathrm{E} u_{\mathrm{m}-1}, \mathrm{E} u_{\mathrm{m}}\right) \\
\leq & \psi\left(\mu_{\mathrm{E}}^{s}\left(u_{\mathrm{m}-1}, z_{\mathrm{m}}\right)\right)=\psi\left(\left[\mathrm{d}\left(u_{\mathrm{m}-1}, \mathrm{E} u_{\mathrm{m}-1}\right)\right]^{\rho_{1}}\right. \\
\cdot & {\left.\left[\mathrm{d}\left(u_{\mathrm{m}}, \mathrm{E} u_{\mathrm{m}}\right)\right]^{\rho_{2}}\right)<\left[\mathrm{d}\left(u_{\mathrm{m}-1}, u_{\mathrm{m}}\right)\right]^{\rho_{1}} } \\
\cdot & {\left[\mathrm{d}\left(u_{\mathrm{m}}, u_{\mathrm{m}+1}\right)\right]^{\rho_{2}} . }
\end{aligned}
\end{aligned}
$$

Due to the above inequality, we obtain

$$
\left(\mathrm{d}\left(u_{\mathrm{m}}, u_{\mathrm{m}+1}\right)\right)^{1-\rho_{2}}<\left(\mathrm{d}\left(u_{\mathrm{m}-1}, u_{\mathrm{m}}\right)\right)^{\rho_{1}},
$$

and from $\rho_{1}+\rho_{2}=1$, we attain that $\mathrm{d}\left(u_{\mathrm{m}}, u_{\mathrm{m}+1}\right)<\mathrm{d}\left(u_{\mathrm{m}-1}\right.$, $u_{\mathrm{m}}$ ) for every $\mathrm{m} \in \mathbb{N}$. Using (31), we get

$$
\mathrm{d}\left(u_{\mathrm{m}}, u_{\mathrm{m}+1}\right) \leq \psi\left(\mathrm{d}\left(u_{\mathrm{m}-1}, u_{\mathrm{m}}\right)\right)
$$

and using previous reasoning

$$
\mathrm{d}\left(u_{\mathrm{m}}, u_{\mathrm{m}+1}\right) \leq \psi^{\mathrm{m}}\left(\mathrm{d}\left(u_{0}, u_{1}\right)\right)
$$

By using the same methods as in the case of $s>0$, we obviously obtain that $\left\{u_{\mathrm{m}}\right\}$ forms a Cauchy sequence in complete metric space. Subsequently, there exists $v \in \mathscr{X}$ such that $\lim _{\mathrm{m} \rightarrow \infty} \mathrm{d}\left(u_{\mathrm{m}}, v\right)=0$, and we claim that this $v$ is a fixed point of E. In case the space $(\mathscr{X}, \mathrm{d})$ is $w$-regular and $\left\{u_{\mathrm{m}}\right\}$ verifies (8), that is, $w\left(u_{\mathrm{m}}, u_{\mathrm{m}+1}\right) \geq 1$ for every $\mathrm{m} \in \mathbb{N}$, we get $w\left(u_{\mathrm{m}}, v\right) \geq 1$. On the other hand, we know (see the proof of case 1) that either

$$
\frac{1}{2} \mathrm{~d}\left(u_{\mathrm{m}}, \mathrm{E} u_{\mathrm{m}}\right) \leq \mathrm{d}\left(u_{\mathrm{m}}, v\right)
$$


or

$$
\frac{1}{2} \mathrm{~d}\left(\mathrm{E} u_{\mathrm{m}}, \mathrm{E}\left(\mathrm{E} u_{\mathrm{m}}\right)\right) \leq \mathrm{d}\left(\mathrm{E} u_{\mathrm{m}}, v\right)
$$

holds, for all $m \in \mathbb{N}$. If (35) is taken, we get

$$
\begin{aligned}
\mathrm{d}\left(u_{\mathrm{m}+1}, \mathrm{E} v\right) & \leq w\left(u_{\mathrm{m}}, v\right) \mathrm{d}\left(\mathrm{E} u_{\mathrm{m}}, \mathrm{E} v\right) \\
& \leq \psi\left(\left[\mathrm{d}\left(u_{\mathrm{m}}, \mathrm{E} u_{\mathrm{m}}\right)\right]^{\rho_{1}} \cdot[\mathrm{d}(v, \mathrm{E} v)]^{\rho_{2}}\right), \\
& =\psi\left(\left[\mathrm{d}\left(u_{\mathrm{m}}, u_{\mathrm{m}+1}\right)\right]^{\rho_{1}} \cdot[\mathrm{d}(v, \mathrm{E} v)]^{\rho_{2}}\right), \\
& <\left[\mathrm{d}\left(u_{\mathrm{m}}, u_{\mathrm{m}+1}\right)\right]^{\rho_{1}} \cdot[\mathrm{d}(v, \mathrm{E} v)]^{\rho_{2}} .
\end{aligned}
$$

If the second condition, (36), is taken, we get

$$
\begin{aligned}
\mathrm{d}\left(u_{\mathrm{m}+2}, \mathrm{E} v\right) & \leq w\left(u_{\mathrm{m}+1}, v\right) \mathrm{d}\left(\mathrm{E}^{2} u_{\mathrm{m}}, \mathrm{E} v\right) \\
& \leq \psi\left(\left[\mathrm{d}\left(\mathrm{E} u_{\mathrm{m}}, \mathrm{E}^{2} u_{\mathrm{m}}\right)\right]^{\rho_{1}} \cdot[\mathrm{d}(v, \mathrm{E} v)]^{\rho_{2}}\right), \\
& =\psi\left(\left[\mathrm{d}\left(u_{\mathrm{m}+1}, u_{\mathrm{m}+2}\right)\right]^{\rho_{1}} \cdot[\mathrm{d}(v, \mathrm{E} v)]^{\rho_{2}}\right), \\
& <\left[\mathrm{d}\left(u_{\mathrm{m}+1}, u_{\mathrm{m}+2}\right)\right]^{\rho_{1}} \cdot[\mathrm{d}(v, \mathrm{E} v)]^{\rho_{2}} .
\end{aligned}
$$

Hence, taking $\mathrm{m} \longrightarrow \infty$ in the last two inequalities, we find that $\mathrm{d}(v, \mathrm{E} v)=0$, that is, $v=\mathrm{E} v$. If the mapping $\mathrm{E}$ is continuous, we obtain $v=\mathrm{E} v$ that we know (see the proof of case 1).

If the last assumption $\left(j_{3}\right)$ holds, as above, we get $\mathrm{E}^{2} v=$ $\lim _{\mathrm{m} \longrightarrow \infty} \mathrm{E}^{2} u_{\mathrm{m}}=\lim _{\mathrm{m} \longrightarrow \infty} u_{\mathrm{m}+2}=v$, and we aim to prove that $\mathrm{E} v=v$. Assuming, on the contrary, that $v \neq \mathrm{E} v$, from

$$
\frac{1}{2} \mathrm{~d}\left(\mathrm{E} v, \mathrm{E}^{2} v\right)=\frac{1}{2} \mathrm{~d}(\mathrm{E} v, v) \leq \mathrm{d}(\mathrm{E} v, v)
$$

using (6), we find that

$$
\begin{aligned}
\mathrm{d}(v, \mathrm{E} v) & =\mathrm{d}\left(\mathrm{E}^{2} v, \mathrm{E} v\right) \leq w(\mathrm{E} v, v) \mathrm{d}\left(\mathrm{E}^{2} v, \mathrm{E} v\right) \\
& \leq \psi\left(\left[\mathrm{d}\left(\mathrm{E} v, \mathrm{E}^{2} v\right)\right]^{\rho_{1}} \cdot\left[\mathrm{d}(v, \mathrm{E} v)^{\rho_{2}}\right]\right) \\
& <[\mathrm{d}(\mathrm{E} v, v)]^{\rho_{1}} \cdot\left[\mathrm{d}(v, \mathrm{E} v)^{\rho_{2}}\right]=\mathrm{d}(\mathrm{E} v, v),
\end{aligned}
$$

a contradiction. As a result, $v=\mathrm{E} v$, that is, $v$ is a fixed point of the mapping $\mathrm{E}$.

Theorem 9. Besides the hypothesis of Theorem 8, if we suppose that $w\left(v, v^{*}\right) \geq 1$ for any $v, v^{*} \in F_{i x}(\mathscr{X})$. Thereupon, $E$ has a unique fixed point in $\mathscr{X}$.

Proof. We shall show that $v$ is a unique fixed point of E. Assume that another $v^{*}$ is fixed point of E, that is, $\mathrm{E} v^{*}=v^{*}$ with $v \neq v^{*}$. In the case that $s>0$, hence, from (6), we have

$$
\begin{gathered}
\frac{1}{2} \mathrm{~d}(v, \mathrm{E} v) \leq \mathrm{d}\left(v, v^{*}\right) \text { implies, } \\
\mathrm{d}\left(v, v^{*}\right) \leq w\left(v, v^{*}\right) \mathrm{d}\left(\mathrm{E} v, \mathrm{E} v^{*}\right) \leq \psi\left(\mu_{\mathrm{E}}^{s}\left(v, v^{*}\right)\right)<\mu_{\mathrm{E}}^{s}\left(v, v^{*}\right) \\
=\left[\rho_{1}\left(\frac{\mathrm{d}(v, \mathrm{E} v) \mathrm{d}\left(v^{*}, \mathrm{E} v^{*}\right)}{d\left(v, v^{*}\right)}\right)^{s}+\rho_{2}\left(\mathrm{~d}\left(v, v^{*}\right)\right)^{s}\right]^{1 / s} .
\end{gathered}
$$

Then,

$$
\mathrm{d}\left(v, v^{*}\right)<\left(\rho_{2}\right)^{1 / s} \mathrm{~d}\left(v, v^{*}\right) \leq \mathrm{d}\left(v, v^{*}\right)
$$

which is a contradiction. In the case that $s=0$, thus, from (6), we get that

$$
0<\mathrm{d}\left(v, v^{*}\right)<0
$$

a contradiction. Consequently, $v=v^{*}$, that is, $\mathrm{E}$ has a unique fixed point in $\mathscr{X}$.

Example 10. Let $\mathscr{X}=[0,2]$ and $d: \mathscr{X} \times \mathscr{X} \longrightarrow[0,+\infty)$ be the usual metric on $\mathbb{R}$ and the mapping $\mathrm{E}: \mathscr{X} \longrightarrow \mathscr{X}$ be determined as

$$
\mathrm{E} u= \begin{cases}\frac{1}{3}, & \text { if } u \in[0,1] \\ \frac{u}{7}, & \text { if } u \in(1,2]\end{cases}
$$

Make allowances for the function $\psi \in \Psi$ with $\psi(t)=t / 3$ and $w: \mathscr{X} \times \mathscr{X} \longrightarrow[0, \infty)$, such that

$$
w(u, z)= \begin{cases}3, & \text { if } u, z \in[0,1] \\ 1, & \text { if } u=0, z=2 \\ 0, & \text { otherwise }\end{cases}
$$

The mapping $\mathrm{E}$ is not continuous, but $\mathrm{E}^{2}$ is a continuous mapping. We choose $\rho_{1}=1 / 5, \rho_{2}=4 / 5$, and $s=2$, then we have

$$
\mu_{\mathrm{E}}^{2}(u, z)=\left[\frac{1}{2}\left(\frac{\mathrm{d}(u, \mathrm{E} u) \mathrm{d}(z, \mathrm{E} z)}{d(u, z)}\right)^{2}+\frac{1}{2}(\mathrm{~d}(u, z))^{2}\right]^{1 / 2}
$$

We obtain that the following cases.

Case 1. For $u, z \in[0,1]$, we get $\mathrm{d}(\mathrm{E} u, \mathrm{E} z)=0$, then (6) holds.

Case 2. For $u=0$ and $z=2$,

$$
\begin{aligned}
& \frac{1}{2} \mathrm{~d}(0, \mathrm{E} 0)=\frac{1}{6}<2=\mathrm{d}(0,2) \Rightarrow, \\
& w(0,2) \mathrm{d}(\mathrm{E} 0, \mathrm{E} 2)=0.04761904761 \leq 0.59780399339 \\
& =\frac{1}{3} \sqrt{\frac{1}{5}\left(\frac{2}{7}\right)^{2}+\frac{4}{5}(2)^{2}} \\
& =\psi \sqrt{\rho_{1}\left(\frac{\mathrm{d}(u, \mathrm{E} u) \mathrm{d}(z, \mathrm{E} z)}{d(u, z)}\right)^{2}+\rho_{2}(\mathrm{~d}(u, z))^{2}} .
\end{aligned}
$$


Other cases are provided, since $w(u, z)=0$. Eventually, the presumptions of Theorem 8 , being satisfied, and $u=1$ / 3 is the fixed point of $\mathrm{E}$.

Corollary 11. Let $(\mathscr{X}, d)$ be a complete metric space and $E$ $: X \longrightarrow X$ a continuous mapping. If there exist $\psi \in \Psi$ such that

$$
\frac{1}{2} d(u, E u) \leq d(u, z) \text { implies } d(E u, E z) \leq \psi\left(\mu_{E}^{s}(u, z)\right),
$$

for each $u, z \in \mathscr{X}$ where $s \geq 0 ; \rho_{i} \geq 0, i=1,2$; and $\rho_{1}+\rho_{2}$ $=1$ with $\rho_{1}<1 / 2$ and $\alpha \in(0,1)$

$$
\mu_{\mathrm{E}}^{s}(u, z)=\left\{\begin{array}{l}
{\left[\rho_{1}\left(\frac{\mathrm{d}(u, \mathrm{E} u) \mathrm{d}(z, \mathrm{E} z)}{d(u, z)}\right)^{s}+\rho_{2}(\mathrm{~d}(u, z))^{s}\right]^{1 / s}, \quad \text { for } s>0, u \neq z,} \\
(\mathrm{~d}(u, \mathrm{E} u))^{\alpha}(\mathrm{d}(z, \mathrm{E} z))^{1-\alpha}, \quad \text { for } s=0, u, z \in \mathscr{X} \backslash \operatorname{Fix}(\mathrm{E}),
\end{array}\right.
$$

therefore, $\mathrm{E}$ has a fixed point in $\mathscr{X}$.

Proof. Case 1: for $s>0$, it follows from Theorem 8, letting $w(u, z)=1$ for $u, z \in \mathscr{X}$.

Case 2: for $s=0$, it follows from Theorem 8, letting $w(u, z)=1$ for $u, z \in \mathscr{X}$ and $\rho_{1}=\alpha, \rho_{2}=1-\alpha$.

Corollary 12. Let $(\mathscr{X}, d)$ be a complete metric space and $E$ $: X \longrightarrow X$ a continuous mapping. If there exist $\lambda \in(0,1)$ such that

$$
\frac{1}{2} d(u, E u) \leq d(u, z) \text { implies } d(E u, E z) \leq \lambda\left(\mu_{E}^{s}(u, z)\right),
$$

for each $u, z \in \mathscr{X}$ where $s \geq 0 ; \rho_{i} \geq 0, i=1,2$; and $\rho_{1}+\rho_{2}$ $=1$ with $\rho_{1}<1 / 2$ and $\alpha \in(0,1)$

$$
\mu_{\mathrm{E}}^{s}(u, z)=\left\{\begin{array}{l}
{\left[\rho_{1}\left(\frac{\mathrm{d}(u, \mathrm{E} u) \mathrm{d}(z, \mathrm{E} z)}{d(u, z)}\right)^{s}+\rho_{2}(\mathrm{~d}(u, z))^{s}\right]^{1 / s}, \quad \text { for } s>0, u \neq z,} \\
(\mathrm{~d}(u, \mathrm{E} u))^{\alpha}(\mathrm{d}(z, \mathrm{E} z))^{1-\alpha}, \quad \text { for } s=0, u, z \in \mathscr{X} \backslash \operatorname{Fix}(\mathrm{E}),
\end{array}\right.
$$

therefore, $\mathrm{E}$ has a fixed point in $\mathscr{X}$.

Proof. Case 1: for $s>0$, it follows from Corollary 11, letting $\psi(v)=\lambda v$ for any $v \geq 0$.

Case 2: for $s=0$, it follows from Corollary 11, letting $\psi$ $(v)=\lambda v$ for any $v \geq 0$ and $\rho_{1}=\alpha, \rho_{2}=1-\alpha$.

Corollary 13. Let $(\mathscr{X}, d)$ be a complete metric space and $E$ $: X \longrightarrow X$ a continuous mapping. If there exist $\lambda \in(0,1)$ such that

$$
\begin{aligned}
\frac{1}{2} d(u, E u) & \leq d(u, z) \text { implies } d(E u, E z) \\
& \leq \lambda(\sqrt{d(u, E u) d(z, E z)})
\end{aligned}
$$
in $\mathscr{X}$.

for each $u, z \in \mathscr{X} \backslash \operatorname{Fix}(\mathrm{E})$; therefore, $\mathrm{E}$ has a fixed point

Proof. For $s=0$, it follows from Corollary 12, letting $\alpha=1 / 2$
Corollary 14. Let $(\mathscr{X}, d)$ be a complete metric space. A continuous mapping $E: X \longrightarrow X$ such that

$$
\begin{aligned}
\frac{1}{2} d(u, E u) & \leq d(u, z) \Rightarrow d(E u, E z) \\
& \leq \alpha \frac{d(u, E u) d(z, E z)}{d(u, z)}+\beta d(u, z), \quad u \neq z,
\end{aligned}
$$

for each $u, z \in \mathscr{X}$, where $\alpha, \beta \in(0,1), \alpha+\beta<1$. Thus, $E$ has a fixed point in $X$.

Proof. For $s=1$, it follows from Corollary 12, letting $\alpha=\lambda \rho_{1}$ and $\beta=\lambda \rho_{2}$ with $\lambda \in(0,1)$ for $u, z \in \mathscr{X}$.

\section{Data Availability}

No data were used to support this study.

\section{Conflicts of Interest}

The authors declare that there is no conflict of interest regarding the publication of this paper. 


\section{Authors' Contributions}

Both authors have contributed equally to this work. Both authors have read and approved the final manuscript.

\section{References}

[1] S. Banach, "Sur les opérations dans les ensembles abstraits et leur application aux équations intégrales," Fundamenta Mathematicae, vol. 3, no. 1, pp. 133-181, 1922.

[2] R. Caccioppoli, "Un teorema generale sullaesistenza di elementi uniti in una trasformazione funzionale," Rendiconti del Seminario Matematico della Università di Padova, vol. 11, pp. 794-799, 1930.

[3] N. Zikria, M. Samreen, T. Kamran, and S. S. Yesilkaya, "Periodic and fixed points for Caristi-type G-contractions in extended b -gauge spaces," Journal of Function Spaces, vol. 2021, Article ID 1865172, 2021.

[4] E. Karapinar, M. De La Sen, and A. Fulga, "A note on the Gornicki-Proinov type contraction," Journal of Function Spaces, vol. 2021, Article ID 6686644, 2021.

[5] D. S. Jaggi, "Some unique fixed point theorems," Indian Journal of Pure and Applied Mathematics, vol. 2, no. 8, pp. 223230, 1977.

[6] E. Karapinar, "Revisiting the Kannan type contractions via interpolation," Advances in the Theory of Nonlinear Analysis and its Application, vol. 2, no. 2, pp. 85-87, 2018.

[7] M. Noorwali, "Common fixed point for Kannan type contractions via interpolation," Journal of Mathematical Analysis, vol. 9, no. 6, pp. 92-94, 2018.

[8] B. Samet, C. Vetro, and P. Vetro, "Fixed point theorems for $\alpha$ - $\psi$-contractive type mappings," Nonlinear Analysis: Theory, Methods \& Applications, vol. 75, no. 4, pp. 2154-2165, 2012.

[9] O. Popescu, "Some new fixed point theorems for $\alpha$-Geraghty contraction type maps in metric spaces," Fixed Point Theory and Applications, vol. 2014, no. 1, 2014.

[10] T. Suzuki, "A generalized Banach contraction principle that characterizes metric completeness," Proceedings of the American Mathematical Society, vol. 136, no. 5, pp. 1861-1869, 2008.

[11] E. O. Austine and D. Igbokwe, "New faster four step iterative algorithm for Suzuki generalized nonexpansive mappings with an application," Advances in the Theory of Nonlinear Analysis and its Application, vol. 5, no. 3, pp. 482-506, 2021.

[12] A. Fulga, "Fixed point theorems in rational form via Suzuki approaches," Results in Nonlinear Analysis, vol. 1, no. 1, pp. 19-29, 2018.

[13] D. R. Babu and G. V. R. Babu, "Fixed points of Suzuki Zcontraction type maps in b-metric spaces," Advances in the Theory of Nonlinear Analysis and its Application, vol. 4, no. 1, pp. 14-28, 2020.

[14] S. S. Yesilkaya, "On interpolative Hardy-Rogers contractive of Suzuki type mappings," Topological Algebra and its Applications, vol. 9, no. 1, pp. 13-19, 2021.

[15] A. Fulga and S. S. Yesilkaya, "On some interpolative contractions of Suzuki type mappings," Journal of Function Spaces, vol. 2021, Article ID 6596096, 2021.

[16] Z. D. Mitrovic, H. Aydi, M. S. M. Noorani, and H. Qawaqneh, "The weight inequalities on Reich type theorem in b-metric spaces," The Journal of Mathematics and Computer Science, vol. 19, no. 1, pp. 51-57, 2019.

[17] E. Karapinar and A. Fulga, "A hybrid contraction that involves Jaggi type,” Symmetry, vol. 11, no. 5, p. 715, 2019.

[18] I. A. Rus, Generalized contractions and applications, Cluj University Press, Clui-Napoca, Romania, 2001. 